LTH 717

NSF-KITP-06-85

hep-ph/0609210

\title{
Anomaly Mediation and Dimensional Transmutation
}

\author{
D.R.T. Jones \\ Dept. of Mathematical Sciences, University of Liverpool, Liverpool L69 3BX, UK \\ and G.G. Ross \\ The Rudolf Peierls Centre for Theoretical Physics, Oxford University, 1 Keble \\ Road, Oxford OX1 3NP, UK
}

\begin{abstract}
We show how a sparticle spectrum characteristic of anomaly mediation can arise from a theory whose Lagrangian contains no explicit mass scale. The scale of supersymmetry breaking is governed by the gravitino mass, which is the vacuum expectation value of the $F$-term of the conformal compensator field, and the tachyonic slepton problem is resolved by the breaking of a $U_{1}^{\prime}$ gauge symmetry at a scale determined by dimensional transmutation.
\end{abstract}




\section{Introduction}

Anomaly mediation is an attractive alternative to the MSUGRA paradigm for low energy supersymmetry [1]- [3]. In Ref. [4] we showed how augmenting the MSSM with an extra $U_{1}$ broken at high energies provides a natural solution to the AMSB tachyonic slepton problem if the extra $U_{1}$ has a Fayet-Iliopoulos term; this model being the explicit construction associated with the scenario first explored in detail in Ref. [5]. Here we show that a similar low energy theory can in fact arise from a scale invariant $M S S M \otimes U_{1}^{\prime}$ theory, with the scale of $U_{1}^{\prime}$ breaking arising by dimensional transmutation, and the only explicit terms of dimension two and three in the Lagrangian being those associated with AMSB. The $U_{1}^{\prime}$ breaking scale also determines the right handed neutrino masses, which in turn determine the observable neutrino masses via the usual see-saw mechanism. The low energy theory differs, however, from that of Ref. [4], in that a light MSSM singlet survives the $U_{1}^{\prime}$ breaking; this raises subtle issues concerning decoupling, as described by Pomarol and Rattazzi (PR) [3].

\section{$2 U(1)^{\prime}$ radiative breaking}

\subsection{The superpotential}

The superpotential for our theory is

$$
W=W_{1}+W_{2}+W_{3},
$$

where $W_{1}$ contains the Yukawa terms responsible for the quark and lepton Dirac masses (including the neutrino Dirac masses):

$$
W_{1}=Q Y_{u} t^{c} H_{2}+Q Y_{d} d^{c} H_{1}+L Y_{e} e^{c} H_{1}+L Y_{\nu} \nu^{c} H_{2}
$$

and $W_{2}$ contains additional terms involving an MSSM singlet sector; a pair of fields $\phi, \bar{\phi}$ charged under $U_{1}^{\prime}$, and gauge singlet fields $U, Z$ :

$$
W_{2}=U\left(\lambda \phi \bar{\phi}-\frac{1}{2} \rho Z^{2}\right)+\frac{1}{6} k U^{3} .
$$

$W_{3}$ contains terms coupling the two sectors, the purpose of which which will be to generate the Higgs $\mu$-term and the right-handed neutrino masses. We will consider two slightly different forms for $W_{3}$ :

$$
W_{3}^{A}=\lambda^{\prime \prime} U H_{1} H_{2}+\frac{1}{2} Y_{\nu^{c}} \phi \nu^{c} \nu^{c}
$$

and

$$
W_{3}^{B}=\lambda^{\prime \prime} Z H_{1} H_{2}+\frac{1}{2} Y_{\nu^{c}} \phi \nu^{c} \nu^{c} .
$$

Here $\lambda, \lambda^{\prime \prime}, \rho, k$ are coupling constants while $Y_{u, d, e, \nu}$ and $Y_{\nu^{c}}$ are $3 \times 3$ matrices in flavour space. The superpotential $W$ is natural in that it contains all possible cubic terms allowed by the symmetry $M S S M \otimes U_{1}^{\prime} \otimes Z_{2}$, where, under $Z_{2}, Z \rightarrow-Z$. and 


\begin{tabular}{|ccccccc|}
\hline$q_{Q}$ & $q_{u^{c}}$ & $q_{d^{c}}$ & $q_{H_{1}}$ & $q_{H_{2}}$ & $q_{\nu^{c}}$ & $q_{\phi}=-q_{\bar{\phi}}$ \\
\hline$-\frac{1}{3} q_{L}$ & $-q_{e}-\frac{2}{3} q_{L}$ & $q_{e}+\frac{4}{3} q_{L}$ & $-q_{e}-q_{L}$ & $q_{e}+q_{L}$ & $-2 q_{L}-q_{e}$ & $4 q_{L}+2 q_{e}$ \\
\hline
\end{tabular}

Table 1: Anomaly free $U_{1}$ symmetry for arbitrary lepton doublet and singlet charges $q_{L}$ and $q_{e}$ respectively.

the remaining fields are invariant (in the case of $W_{3}^{A}$ ) or arranged so that $H_{1} H_{2} \rightarrow$ $-H_{1} H_{2}$ (in the case of $W_{3}^{B}$ ).

Note that $W$ contains no explicit mass scale. The electroweak scale (and the associated supersymmetry-breaking scale) are generated via anomaly mediation, while the $U_{1}^{\prime}$-breaking scale is generated by dimensional transmutation, as we shall discuss.

The $U_{1}^{\prime}$ charge assignments of the various fields are shown in Table 1.

\subsection{The scalar potential}

Our theory is defined by the superpotential Eq. (11), with the addition of anomaly mediation soft-breaking terms of the general form

$$
\begin{aligned}
L_{\mathrm{SOFT}} & =\sum_{\phi} m_{\phi}^{2} \phi^{*} \phi+\left[m_{3}^{2} H_{1} H_{2}+\sum_{i=1}^{3} \frac{1}{2} M_{i} \lambda_{i} \lambda_{i}+\text { h.c. }\right] \\
& +\left[H_{2} Q h_{t} t^{c}+H_{1} Q h_{b} b^{c}+H_{1} L h_{\tau} \tau^{c}+\text { h.c. }\right]
\end{aligned}
$$

where

$$
\begin{aligned}
M_{i} & =m_{0} \beta_{g_{i}} / g_{i} \\
h_{Y} & =-m_{0} \beta_{Y} \\
m_{\phi}^{2} & =\frac{1}{2} m_{0}^{2} \mu \frac{d}{d \mu} \gamma_{\phi} .
\end{aligned}
$$

Here $m_{0}$ is the gravitino mass, $\beta_{g_{i}}$ are the gauge $\beta$-functions, $\gamma$ the chiral supermultiplet anomalous dimension, $Y$ stands for any Yukawa coupling and $\beta_{Y_{t, b, \tau}}$ are the Yukawa $\beta$-functions. (We will discuss presently the origin of the Higgs $\mu$-term and associated soft breaking term).

Let us seek an extremum of the scalar potential such that $\phi, \bar{\phi}, Z$ obtain vacuum expectation values which are of the same order and much bigger than $m_{0}$. We therefore begin by writing down the scalar potential corresponding to the superpotential $W_{2}$. Note that this superpotential has no accidental additional continuous symmetries; consequently at the extremum there will be no pseudo-Goldstone bosons. The relevant tree potential may be written

$$
\begin{aligned}
V & =\frac{1}{2} m_{1}^{2} x^{2}+\frac{1}{2} m_{2}^{2} y^{2}+\frac{1}{2} m_{3}^{2} z^{2}+\frac{1}{2} m_{u}^{2} u^{2}+h_{1} u x y+\frac{1}{2} h_{2} u z^{2}+\frac{1}{6} h_{3} u^{3} \\
& +\frac{1}{4} \lambda^{2}\left(x y-z^{2}+\frac{1}{2} \bar{k} u^{2}\right)^{2}+\frac{1}{4} \lambda^{2} u^{2}\left(x^{2}+y^{2}\right)+\frac{1}{2} \lambda \rho u^{2} z^{2}+\frac{1}{8} g_{\phi}^{2}\left(x^{2}-y^{2}\right)^{2}
\end{aligned}
$$


Here $g_{\phi}=q_{\phi} g^{\prime}$ where $g^{\prime}$ is the $U_{1}^{\prime}$ coupling, $x / \sqrt{2}=\langle\phi\rangle, y / \sqrt{2}=\langle\bar{\phi}\rangle, z=\sqrt{\frac{\rho}{\lambda}}\langle Z\rangle$, $u / \sqrt{2}=\langle U\rangle, m_{\phi}=m_{1}, m_{\bar{\phi}}=m_{2}, m_{3}^{2}=2 m_{Z}^{2} \lambda / \rho$ and $\bar{k}=k / \lambda$. The cubic couplings $h_{1,2,3}$ are related to $\beta_{\lambda, \rho, k}$ according to Eq. (7b):

$$
\begin{aligned}
& h_{1}=\frac{1}{\sqrt{2}} h_{\lambda}=-\frac{m_{0}}{\sqrt{2}} \beta_{\lambda} \\
& h_{2}=-\frac{\sqrt{2} \lambda}{\rho} h_{\rho}=\frac{\sqrt{2} \lambda m_{0}}{\rho} \beta_{\rho} \\
& h_{3}=\frac{1}{\sqrt{2}} h_{k}=-\frac{m_{0}}{\sqrt{2}} \beta_{k} .
\end{aligned}
$$

For an extremum we have

$$
\begin{aligned}
x\left[m_{1}^{2}+\frac{1}{2} g_{\phi}^{2}\left(x^{2}-y^{2}\right)+\frac{1}{2} \lambda^{2} u^{2}\right]+y\left[\frac{1}{2} \lambda^{2}\left(x y-z^{2}+\frac{\bar{k}}{2} u^{2}\right)+h_{1} u\right] & =0 \\
y\left[m_{2}^{2}-\frac{1}{2} g_{\phi}^{2}\left(x^{2}-y^{2}\right)+\frac{1}{2} \lambda^{2} u^{2}\right]+x\left[\frac{1}{2} \lambda^{2}\left(x y-z^{2}+\frac{\bar{k}}{2} u^{2}\right)+h_{1} u\right] & =0 \\
z\left[m_{3}^{2}-\lambda^{2}\left(x y-z^{2}+\frac{\bar{k}}{2} u^{2}\right)+\lambda \rho u^{2}+h_{2} u\right] & =0 \\
u\left[m_{u}^{2}+\frac{1}{2} \lambda^{2}\left(x^{2}+y^{2}\right)+\frac{1}{2} \bar{k} \lambda^{2}\left(x y-z^{2}+\frac{\bar{k}}{2} u^{2}\right)+\lambda \rho z^{2}\right] & \\
+h_{1} x y+\frac{1}{2} h_{2} z^{2}+\frac{1}{2} h_{3} u^{2} & =0 .
\end{aligned}
$$

We seek an an extremum such that the $U_{1}^{\prime}$ is broken at a scale much larger than $m_{0}$. For this to be a minimum, we see from Eq. (8) that it must correspond to $x \sim y \sim z>>u$. Then, setting $x \approx y \approx z \approx M$, we have from Eq. (10d) that

$$
u \approx-\frac{2 h_{1}+h_{2}}{2\left(\lambda^{2}+\lambda \rho\right)}
$$

so that $u$ is naturally of $O\left(\frac{m_{0}}{16 \pi^{2}}\right)$. Now in anomaly mediation we have (for a typical $\mathrm{TeV}$ sparticle spectrum) $m_{0} \sim 40 \mathrm{TeV}$; we thus obtain, in the case $W_{3} \equiv W_{3}^{A}$, a contribution to the Higgs $\mu$-term from Eqs. (41), (11) of appropriate magnitude (around $0.5 \mathrm{TeV}$ ) without having to assume a small value of the coupling $\lambda^{\prime \prime}$. It is also easy to show (from Eqs. (10a), (10b) ) that (to leading order in $M$ )

$$
x^{2}-y^{2}=\frac{m_{2}^{2}-m_{1}^{2}}{g_{\phi}^{2}},
$$

so that the contribution to the slepton doublet mass is simply

$$
\Delta m_{L}^{2}=\frac{1}{2} g_{\phi}^{2} \frac{q_{L}}{q_{\phi}}\left(x^{2}-y^{2}\right)=\frac{m_{2}^{2}-m_{1}^{2}}{2} \frac{q_{L}}{q_{\phi}}
$$

(with a similar formula for the slepton singlet mass) so, as long as we pick charges such that $q_{L}$ and $q_{e}$ have the same sign (depending on the sign of $m_{2}^{2}-m_{1}^{2}$ ), we have a potential solution to the tachyonic slepton problem. Since $m_{1,2}^{2}$ depend on unknown couplings we can write

$$
\frac{m_{2}^{2}-m_{1}^{2}}{2 q_{\phi}}=\xi
$$


where $\xi$ is an effective Fayet-Iliopoulos parameter.

Setting $x=R \cos \Omega$ and $y=R \sin \Omega$, we find also from Eqs. (10a), (10b) that

$$
\sin 2 \Omega=-\frac{m_{3}^{2}+\left(2 h_{1}+h_{2}\right) u+\lambda \rho u^{2}}{m_{1}^{2}+m_{2}^{2}+\lambda^{2} u^{2}} .
$$

Now our desired extremum corresponds to $\Omega \approx \pi / 4$; we see easily from Eq. (15) and using Eq. (11) that at this extremum we require

$$
\Delta=m_{1}^{2}+m_{2}^{2}+m_{3}^{2}-\frac{\left(h_{1}+\frac{1}{2} h_{2}\right)^{2}}{\lambda(\lambda+\rho)}=0+O\left[\left(m_{0}^{4} / M^{2}\right)\right] .
$$

Now this looks like a fine-tuning of the fundamental parameters; however when we bear in mind that all these parameters are functions of scale then this interpretation changes. The crucial point is that if we are interested in the effective potential of a set of fields at an (approximately common) value of these fields much larger than any explicit mass parameters, then the appropriate scale to evaluate the potential is one equal to the the scale set by the value of the fields themselves. This simply minimises the effect of radiative corrections on the tree potential.

Now from Eq. (8) it is clear that for $m_{1}^{2}+m_{2}^{2}+m_{3}^{2} \leq 0$, the potential $V$ is unbounded from below as for $u=0$ and $x=y=z \rightarrow \infty$. Crucially, however, it is quite natural to have $\Delta \leq 0$ at some scale (corresponding to $\mu<M$ ) and then $\Delta>0$ for $\mu>M$. We will demonstrate that this mechanism works explicitly in the next section by examining the variation of $\Delta$ with renormalisation scale. It follows that the potential will develop a minimum at the scale such that Eq. (16) is satisfied. It is easy to show from Eq. (8) that in the neighbourhood of the minimum,

$$
V=\frac{1}{2} \Delta M^{2}+O\left[m_{0}^{4}\right] .
$$

This is, of course, an example of dimensional transmutation as originally described by Coleman and Weinberg [6]; the relation Eq. (16) is analogous to the relation CW wrote down between the quartic scalar and gauge couplings (valid at the extremum of the potential) in massless scalar QED. In describing the breaking of a gauge symmetry at high energies our approach here is reminiscent of Witten's inverted hierarchy model [7.

The upshot of all this is that without fine-tuning, our scale invariant potential can quite naturally have an extremum such that $x \sim y \sim z \sim M>>m_{1,2,3}$, thus achieving $U_{1}^{\prime}$ breaking in a manner similar to that envisaged in Ref. [4, but without the explicit introduction of the breaking scale in the form of an FI term. 


\subsection{The anomalous dimensions}

At one loop the anomalous dimensions of the various fields which only appear in $W_{2}$ and $W_{3}$ are given by (for $W_{3} \equiv W_{3}^{A}$ )

$$
\begin{aligned}
16 \pi^{2} \gamma_{\phi} & =\lambda^{2}+\frac{1}{2} \operatorname{Tr}\left(Y_{\nu^{c}}\right)^{2}-2 g^{\prime 2} q_{\phi}^{2} \\
16 \pi^{2} \gamma_{\bar{\phi}} & =\lambda^{2}-2 g^{\prime 2} q_{\phi}^{2} \\
16 \pi^{2} \gamma_{U} & =\lambda^{2}+\frac{1}{2} \rho^{2}+\frac{1}{2} k^{2}+2 \lambda^{\prime \prime 2} \\
16 \pi^{2} \gamma_{Z} & =\rho^{2}
\end{aligned}
$$

while for $W_{3} \equiv W_{3}^{B}$, Eqs. (18c) and (18d) are replaced by

$$
\begin{aligned}
& 16 \pi^{2} \gamma_{U}=\lambda^{2}+\frac{1}{2} \rho^{2}+\frac{1}{2} k^{2} \\
& 16 \pi^{2} \gamma_{Z}=\rho^{2}+2 \lambda^{\prime \prime 2}
\end{aligned}
$$

For the MSSM non-singlet fields and $\nu^{c}$ we have

$$
\begin{aligned}
16 \pi^{2}\left(\gamma_{L}\right)_{j}^{i} & =\left(Y_{e}\right)^{i k}\left(Y_{e}\right)_{j k}+\left(Y_{\nu}\right)^{i k}\left(Y_{\nu}\right)_{j k}-2 C_{H} \delta_{j}^{i}, \\
16 \pi^{2}\left(\gamma_{e^{c}}\right)_{j}^{i} & =\left(Y_{e}\right)^{k i}\left(Y_{e}\right)_{k j}-2 C_{e^{c}} \delta_{j}^{i}, \\
16 \pi^{2}\left(\gamma_{\nu^{c}}\right)_{j}^{i} & =\left(Y_{\nu^{c}}\right)^{i k}\left(Y_{\nu^{c}}\right)_{j k}+2\left(Y_{\nu}\right)^{k i}\left(Y_{\nu}\right)_{k j}-2 g^{\prime 2} q_{\nu^{c}}^{2} \delta_{j}^{i} \\
16 \pi^{2}\left(\gamma_{Q}\right)_{j}^{i} & =\left(Y_{d}\right)^{i m}\left(Y_{d}\right)_{j m}+\left(Y_{u}\right)^{i m}\left(Y_{u}\right)_{j m}-2 C_{Q} \delta_{j}^{i}, \\
16 \pi^{2}\left(\gamma_{d^{c}}\right)_{j}^{i} & =2\left(Y_{d}\right)^{m i}\left(Y_{d}\right)_{m j}-2 C_{d^{c}} \delta_{j}^{i}, \\
16 \pi^{2}\left(\gamma_{u^{c}}\right)_{j}^{i} & =2\left(Y_{u}\right)^{m i}\left(Y_{u}\right)_{m j}-2 C_{u^{c}} \delta_{j}^{i}, \\
16 \pi^{2} \gamma_{H_{1}} & =3\left(Y_{d}\right)^{i j}\left(Y_{d}\right)_{i j}+\left(Y_{e}\right)^{i j}\left(Y_{e}\right)_{i j}+\lambda^{\prime \prime 2}-2 C_{H}, \\
16 \pi^{2} \gamma_{H_{2}} & =3\left(Y_{u}\right)^{i j}\left(Y_{u}\right)_{i j}+\left(Y_{\nu}\right)^{i j}\left(Y_{\nu}\right)_{i j}+\lambda^{\prime \prime 2}-2 C_{H},
\end{aligned}
$$

where

$$
\begin{aligned}
C_{Q} & =\frac{4}{3} g_{3}^{2}+\frac{3}{4} g_{2}^{2}+\frac{1}{60} g_{1}^{2}+q_{Q}^{2} g^{\prime 2} \\
C_{L} & =\frac{3}{4} g_{2}^{2}+\frac{3}{20} g_{1}^{2}+q_{L}^{2} g^{\prime 2} \\
C_{u^{c}} & =\frac{4}{3} g_{3}^{2}+\frac{4}{15} g_{1}^{2}+q_{u^{c}}^{2} g^{\prime 2} \\
C_{d^{c}} & =\frac{4}{3} g_{3}^{2}+\frac{1}{15} g_{1}^{2}+q_{d^{c}}^{2} g^{\prime 2} \\
C_{e^{c}} & =\frac{3}{5} g_{1}^{2}+q_{e}^{2} g^{\prime 2} \\
C_{H} & =\frac{3}{4} g_{2}^{2}+\frac{3}{20} g_{1}^{2}+q_{H}^{2} g^{\prime 2}
\end{aligned}
$$

and we have written $q_{H}^{2}=q_{H_{1}}^{2}=q_{H_{2}}^{2}$, since $q_{H_{1}}=-q_{H_{2}}$.

\subsection{RG evolution and radiative breaking}

In this section we work in the simplified approximation where we neglect $\lambda^{\prime \prime}$ and $Y_{\nu}$. As a result the renormalisation group evolution equations of $\lambda, Y_{\nu^{c}}, \rho k$ and $g^{\prime}$ become (at one loop) a closed system, which simplifies the analysis, and makes it 
the same whether we use $W_{3}^{A}$ or $W_{3}^{B}$. Moreover we will assume a simplified form for $Y_{\nu^{c}}$, to wit

$$
Y_{\nu^{c}}=\left(\begin{array}{ccc}
0 & 0 & 0 \\
0 & 0 & 0 \\
0 & 0 & \lambda^{\prime}
\end{array}\right) .
$$

The $\beta$-functions for the Yukawa couplings are then:

$$
\begin{aligned}
16 \pi^{2} \beta_{\lambda} & =\lambda\left(\gamma_{\phi}+\gamma_{\bar{\phi}}+\gamma_{U}\right) \\
& =\lambda\left[3 \lambda^{2}+\frac{1}{2} \lambda^{\prime 2}+\frac{1}{2} \rho^{2}+\frac{1}{2} k^{2}-4 g^{\prime 2} q_{\phi}^{2}\right] \\
16 \pi^{2} \beta_{\lambda^{\prime}} & =\lambda^{\prime}\left(\gamma_{\phi}+2 \gamma_{\nu^{c}}\right) \\
& =\lambda^{\prime}\left[\lambda^{2}+\frac{5}{2} \lambda^{\prime 2}-2 g^{\prime 2}\left(q_{\phi}^{2}+2 q_{\nu^{c}}^{2}\right)\right] \\
16 \pi^{2} \beta_{\rho} & =\rho\left(\gamma_{U}+2 \gamma_{Z}\right)=\rho\left[\lambda^{2}+\frac{5}{2} \rho^{2}+\frac{1}{2} k^{2}\right] \\
16 \pi^{2} \beta_{k} & =3 k \gamma_{U}=3 k\left[\lambda^{2}+\frac{1}{2} \rho^{2}+\frac{1}{2} k^{2}\right] .
\end{aligned}
$$

The $\beta$-function for the $U_{1}^{\prime}$ gauge coupling is

$$
16 \pi^{2} \beta_{g^{\prime}}=b^{\prime} g^{\prime 3}
$$

where

$$
b^{\prime}=3\left(q_{e}^{2}+3\left(q_{u^{c}}^{2}+q_{d^{c}}^{2}\right)+6 q_{Q}^{2}+2 q_{L}^{2}+q_{\nu^{c}}^{2}\right)+2\left(q_{H_{1}}^{2}+q_{H_{2}}^{2}\right)+2 q_{\phi}^{2} .
$$

We then find using the AMSB mass formula

$$
\left(m^{2}\right)^{i}{ }_{j}=\frac{1}{2} m_{0}^{2} \mu \frac{d}{d \mu} \gamma_{j}^{i}
$$

that

$$
\begin{aligned}
16 \pi^{2} m_{\phi^{2}} & =\frac{1}{2} m_{0}^{2}\left(2 \lambda \beta_{\lambda}+\lambda^{\prime} \beta_{\lambda^{\prime}}-4 g^{\prime} \beta_{g^{\prime}} q_{\phi}^{2}\right) \\
16 \pi^{2} m_{\bar{\phi}^{2}} & =\frac{1}{2} m_{0}^{2}\left(2 \lambda \beta_{\lambda}-4 g^{\prime} \beta_{g^{\prime}} q_{\phi}^{2}\right) \\
16 \pi^{2} m_{Z}^{2} & =\frac{1}{2} m_{0}^{2}\left(2 \rho \beta_{\rho}\right) .
\end{aligned}
$$

Substituting in Eq. (16) we thus have

$$
\begin{aligned}
\left(16 \pi^{2}\right)^{2} \frac{\Delta}{m_{0}^{2}} & =\left[32 \lambda^{5}+16 \lambda^{2} k^{2} \rho+8 \lambda \rho^{2} k^{2}+64 \lambda^{4} \rho+4 \lambda^{3} \lambda^{\prime 2}+56 \lambda^{3} \rho^{2}\right. \\
& +48 \lambda^{2} \rho^{3}+8 \lambda^{3} k^{2}+12 \lambda^{2} \lambda^{\prime 2} \rho+9 \lambda^{\prime 4} \lambda+10 \lambda^{\prime 4} \rho+24 \lambda \rho^{4}+8 \lambda \lambda^{\prime 2} \rho^{2} \\
& -g^{\prime 2}\left(1024 \lambda^{2} q_{L}^{2} \rho+256 \lambda^{2} q_{e}^{2} \rho-64 \lambda^{\prime 2} q_{L}^{2} \lambda+192 \lambda^{\prime 2} q_{L}^{2} \rho-16 \lambda^{\prime 2} q_{e}^{2} \lambda\right. \\
& +48 \lambda^{\prime 2} q_{e}^{2} \rho+1024 \lambda q_{L}^{2} \rho^{2}+256 \lambda q_{e}^{2} \rho^{2}+1024 \lambda^{2} q_{L} q_{e} \rho-64 \lambda^{\prime 2} q_{L} q_{e} \lambda \\
& \left.+192 \lambda^{\prime 2} q_{L} q_{e} \rho+1024 \lambda q_{L} q_{e} \rho^{2}\right)-g^{\prime 4}\left(4608 q_{e}^{4} \rho+55296 q_{L}^{4} \lambda\right. \\
& +38912 q_{L}^{4} \rho+5632 q_{e}^{4} \lambda+97792 q_{e}^{2} q_{L}^{2} \lambda+73216 q_{e}^{2} q_{L}^{2} \rho+37888 q_{e}^{3} q_{L} \lambda \\
& \left.\left.+29696 q_{e}^{3} q_{L} \rho+116736 q_{L}^{3} q_{e} \lambda+83968 q_{L}^{3} q_{e} \rho\right)\right] /(8(\lambda+\rho)] .
\end{aligned}
$$




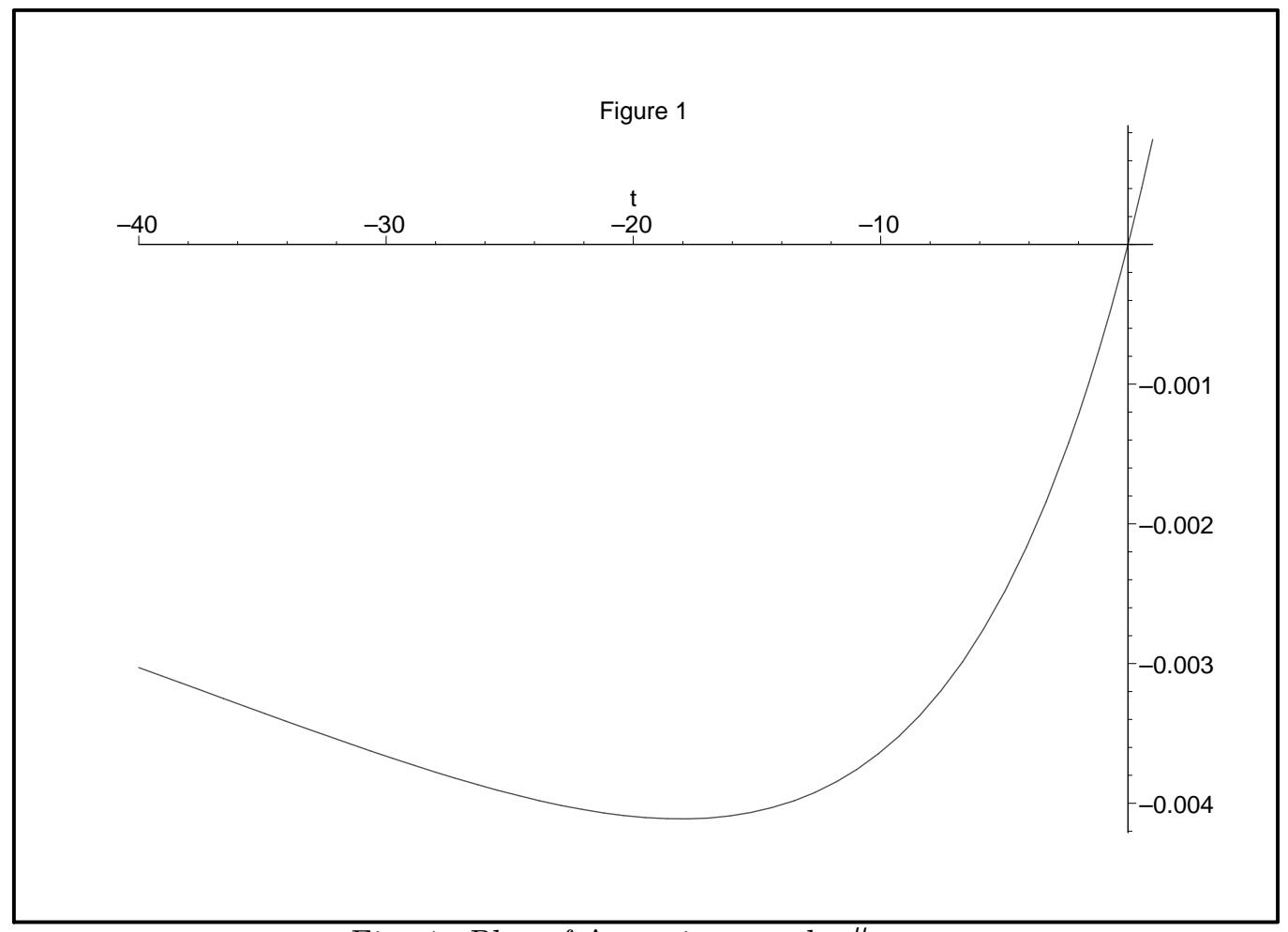

Fig. 1: Plot of $\Delta$ against $t=\ln \frac{\mu}{\mu_{0}}$

In Fig. 1 we plot $\Delta$ against $t=\ln \frac{\mu}{\mu_{0}}$, choosing initial values so that $\Delta=0$ when $\mu=\mu_{0}$. (Specifically, we have taken, at $\mu=\mu_{0}, \rho=\lambda / 10, \lambda^{\prime}=k=2 \lambda, \lambda=0.4784$, $g^{\prime}=1, \xi=1 \mathrm{TeV}^{2}$ and $q_{L}=q_{e}=0.08$. We see that $\Delta$ indeed has the desired behaviour, with $\Delta<0$ at $\mu<\mu_{0}$ to $\Delta>0$ at $\mu>\mu_{0}$. Clearly the potential will have a minimum for $\mu \sim \mu_{0}$.

\section{The low energy theory}

\subsection{The Higgs spectrum}

If we take the superpotential Eq. (3) and shift the fields by their vacuum expectation values $\left(U \rightarrow u / \sqrt{2}+U, \phi \rightarrow x / \sqrt{2}+\phi, \bar{\phi} \rightarrow y / \sqrt{2}+\bar{\phi}, Z \rightarrow \sqrt{\frac{\lambda}{\rho}} z+Z\right)$, it is easy to see that, to $O\left(\frac{u}{x}\right)$, the linear combination

$$
H=\frac{1}{R^{\prime}}\left[x \bar{\phi}+y \phi-\sqrt{\frac{2 \rho}{\lambda}} z Z\right]
$$

where

$$
R^{\prime 2}=x^{2}+y^{2}+\frac{2 \rho}{\lambda} z^{2}
$$


combines with $U$ so that both get large supersymmetric mass terms. The combination

$$
G=\frac{1}{R}[y \bar{\phi}-x \phi]
$$

where

$$
R^{2}=x^{2}+y^{2}
$$

is the Higgs/Goldstone multiplet, combining with the $U_{1}^{\prime}$ gauge multiplet to produce a spin 1 massive supermultiplet. The orthogonal combination

$$
L=\frac{1}{R^{\prime \prime}}\left[x \bar{\phi}+y \phi+\sqrt{\frac{\lambda}{2 \rho}} \frac{x^{2}+y^{2}}{z} Z\right]
$$

where

$$
R^{\prime \prime 2}=x^{2}+y^{2}+\frac{\lambda}{2 \rho} \frac{\left(x^{2}+y^{2}\right)^{2}}{z^{2}}
$$

has a large vacuum expectation value but obtains a mass of order $u$, which from Eq. (11) is of order TeV. Examined in more detail, we find that the two scalar components of $L$ have masses of $O(u \sqrt{\rho})$ and its fermionic component a mass of order $u \rho$ if, as we shall motivate later, we choose $\rho<<\lambda$. It is interesting to note that the $U H_{1} H_{2}$ or $Z H_{1} H_{2}$ terms in the alternative superpotential terms $W_{3}^{A}$ or $W_{3}^{B}$ of Eqs. (41) and (51) means that part of our theory resembles the NMSSM. However, unlike the NMSSM, the light singlet state decouples to $O\left(\frac{u}{x}\right)$ or almost decouples from the MSSM fields, in the first case because $U$ has no $L$ component while in the latter case the coefficient of the trilinear term must be chosen to be very small for phenomenological reasons. In both cases however the existence of the light field causes a phenomenon of AMSB non-decoupling, described by Pomarol and Rattazzi (PR) [3], which effects the low energy mass spectrum as discussed below.

\subsection{The Higgs $\mu$-term and $b$-term}

\subsubsection{A fine tuned solution}

Let us consider first the case $W_{3} \equiv W_{3}^{A}$ (Eq. (4)). The vacuum described in Section 2.2 clearly generates a Higgs $\mu$-term $\mu_{1}=\lambda^{\prime \prime}\langle U\rangle$ which is naturally of the right order of magnitude. It also, however, generates a soft breaking Higgs $b$-term of the form $b_{1}=m_{0} \mu_{1}$ which is too large; a generic problem with the scenario which has been noticed by a number of authors.

There is, however another Weyl invariant operator which generates both $\mu$-term and a $b$-term, to wit

$$
\delta L=\sigma \int d^{4} \theta \frac{\Phi^{\dagger}}{\Phi} H_{1} H_{2}
$$

where $\Phi=1+m_{0} \theta^{2}$ is the conformal compensator field. This gives $\mu_{2}=\sigma m_{0}$ and $b_{2}=-m_{0} \mu_{2}$. Thus we have $\mu=\mu_{1}+\mu_{2}$ and $b=m_{0}\left(\mu_{1}-\mu_{2}\right)$. It is clear that if we proceed to determine $\mu$ and $b$ via the electroweak minimisation conditions in the customary way then this amounts to a degree of fine-tuning in order to achieve $b \sim \mu^{2}$ 


\subsubsection{An unnatural solution}

PR [3] propose an ingenious solution to this problem as follows. They introduce the further contribution to the superpotential

$$
W_{4}=\lambda^{\prime \prime} S H_{1} H_{2}+\frac{1}{6} k^{\prime} S^{3}+\frac{1}{2} k^{\prime \prime} Z S^{2} .
$$

The superpotential $W_{4}$ produces at one loop kinetic mixing of $Z$ and $S$ :

$$
\delta L \sim k^{\prime} k^{\prime \prime} \int d^{4} \theta Z^{\dagger} S
$$

The large vev for $Z$ produces a large supersymmetric mass term for $S$, so that it decouples at low energies and can be eliminated via its equation of motion which for small $k^{\prime}$ is

$$
S=-k^{\prime \prime} \frac{H_{1} H_{2}}{Z}
$$

giving rise to a contribution to the effective low energy theory of the form

$$
\delta L \sim \int d^{4} \theta \frac{Z^{\dagger}}{Z} H_{1} H_{2}
$$

and hence via anomaly mediation a contribution to the $\mu$ term $\mu=\mu_{3}$ and an associated contribution to the $b$-term of the form

$$
b_{3}=-m_{0} \beta_{\mu_{3}} .
$$

Thus one achieves a $b$ of the right order of magnitude (relative to $\mu_{3}$ ). Then if $\lambda^{\prime \prime}$ and $\rho$ were sufficently small that the contributions to $b$ associated with them were of similar size to $b_{3}$ then we would have $\mu \sim \mu_{3}$ and $b \sim b_{1}+b_{2}+b_{3}$, so that once more we can determine $\mu, b$ via electroweak minimisation. (If we assume that the $\mathrm{PR}$ mechanism is the only source of $\mu, b$ then it is difficult to achieve the electroweak vacuum [5], because Eq. (39) would mean $\mu$ and $b$ were not independent.)

The problem with this scenario is that in order to write down the superpotential $W_{4}$ we must abandon the $Z \rightarrow-Z$ symmetry which rendered our theory natural. This is because the simultaneous presence of the $S^{3}$ term and the $Z S^{2}$ term requires $Z$ and $S$ have the same symmetry properties. As a result a natural theory would also allow additional terms in Eq. (3) of the form $Z^{3}$ and $Z^{2} S$ which unfortunately spoil the mechanism proposed by PR.

\subsubsection{A natural solution without fine tuning}

Here we propose a very simple mechanism which does not involve additional fields to generate the $\mu$ and $b$ terms of the correct magnitude in a way consistent with the $Z_{2}$ symmetry. This is achieved through the case $W_{3} \equiv W_{3}^{B}$ (Eq. (5)).

The $U_{1}^{\prime}$-breaking proceeds exactly as before and so from Eq. (5) we obtain a $\mu$ term of the form $\mu=\lambda^{\prime \prime}\langle Z\rangle$. Since $\langle Z\rangle$ is of order the $U_{1}^{\prime}$ breaking scale it is therefore necessary to assume that $\lambda^{\prime \prime}$ is very small. We will return to this point presently. 
The reason that we can achieve a suitable $b$-term is that since

$$
Z=\frac{1}{\sqrt{R^{\prime 2}+R^{\prime \prime 2}}}\left(-R^{\prime} H+R^{\prime \prime} L\right)
$$

we can, c.f. Eqs. (30) and (33), arrange by making $\rho \ll \lambda$ that $Z \sim L$. This will suppress the $B$ term since while $F_{H}=\langle H\rangle m_{0}, F_{L}=0$. Quantitatively we have

$$
b=\sqrt{2} \frac{\rho}{\lambda} \mu m_{0} .
$$

Thus we retain naturalness by avoiding the need for cancellation between distinct contributions to $b$. Although we do require two dimensionless couplings ( $\rho$ and $\lambda^{\prime \prime}$ ) to be small this can be achieved by, for example, the Froggatt-Nielsen mechanism, generating these terms through higher dimension terms suppressed by a large messenger mass.

\subsection{The Low Energy Theory}

The existence of a light singlet field, $L$, introduces additional contributions to the soft supersymmetry breaking terms due to the non-decoupling phenomenon discussed in PR [3]. Following PR we have, in place of Eqs. (7ad), (7b) and (7c), the equations

$$
\begin{aligned}
M_{i} & =m_{0} \beta_{g_{i}} / g_{i} \\
h_{Y} & =-m_{0}\left(\frac{\partial}{\partial \ln \mu}+\frac{\partial}{\partial \ln M}\right) \ln Z_{Y}(\mu, M) \\
m_{\phi}^{2} & =-\frac{1}{2} m_{0}^{2}\left(\frac{\partial}{\partial \ln \mu}+\frac{\partial}{\partial \ln M}\right)^{2} \ln Z_{Y}(\mu, M),
\end{aligned}
$$

where $M$ is the $U(1)^{\prime}$ breaking scale and $\mu$ is the low-energy scale parameter $(\mu \ll$ $M)$. The $\mu$ dependent terms are the normal anomaly mediated terms while the $M$ dependent terms are the non-decoupling terms due to the light scalar. These equations apply in the limit $\rho \ll \lambda$ where $F_{L}=0$. One sees that the effect of these non-decoupling terms is simply to retain the contributions of the $U_{1}^{\prime}$ gauge boson to the soft breaking terms even though the $U_{1}^{\prime}$ gauge coupling freezes out at the breaking scale $M$. Thus for example we have for the soft $Q t^{c} H_{2}$ coupling, $h_{t}$,

$$
16 \pi^{2} h_{t}=-m_{0} \lambda_{t}\left(6 \lambda_{t}^{2}-2\left(C_{H}+C_{Q}+C_{t^{c}}\right)\right)
$$

where we have for simplicity retained only the top-quark Yukawa coupling. The gauge pieces $C_{Q}$ etc. are as given in Eq. (21), but the $U_{1}^{\prime}$ coupling is to be evaluated at the $U_{1}^{\prime}$ breaking scale.

\subsection{Phenomenological implications}

For both the cases $W_{3} \equiv W_{3}^{A}$ and $W_{3} \equiv W_{3}^{B}$, in the limit $g^{\prime} \rightarrow 0$, the additional soft terms due to non-decoupling associated with the light scalar state are negligible 
and, to a good approximation, we recover the spectrum of Ref. [4 for the MSSM states. This is because, as discussed earlier, the coupling of the light singlet $L$ to the Higgs is very small. The fermionic component, $\widetilde{L}$, of $L$ will, however be the LSP. The accelerator lower limits of around $46 \mathrm{GeV}$ will not apply because of the weak coupling of the state; in our favoured scenario for Higgs $\mu$-term generation we estimate its mass to be around $10 \mathrm{GeV}$. The cosmological and phenomenological

implications of a $\widetilde{L}$ LSP are similar to that of a gravitino LSP and will be discussed in detail elsewhere.

For the case when the new gauge interaction is not negligible there will be additional supersymmetry breaking soft contributions to the MSSM states. We will also return elsewhere to a consideration of the phenomenology in this case too.

\section{Acknowledgements}

Both DRTJ and GGR visited CERN and KITP (Santa Barbara) while part of this work was done. This work was partially supported by the EC 6th Framework Programme MRTN-CT-2004-503369, and by the National Science Foundation under Grant No. PHY99-07949.

\section{References}

[1] L. Randall and R. Sundrum, Nucl. Phys. B 557 (1999) 79

[2] G.F. Giudice et al, JHEP 9812 (1998) 27

[3] A. Pomarol and R. Rattazzi, JHEP 9905 (1999) 013

[4] R. Hodgson, I. Jack, D.R.T. Jones and G.G. Ross, Nucl. Phys. B 728 (2005) 192

[5] I. Jack and D.R.T. Jones, Phys. Lett. B 482 (2000) 167

[6] S.R. Coleman and E. Weinberg, Phys. Rev. D 7 (1973) 1888

[7] E. Witten, Phys. Lett. B 105 (1981)267 